\title{
Epidural Spinal Canal Space
}

National Cancer Institute

\section{Source}

National Cancer Institute. Epidural Spinal Canal Space. NCI Thesaurus. Code C41449.

Space between the dura mater and the walls of the vertebral canal. 